\title{
A novel hepatokine, HFREP1, plays a crucial role in the development of insulin resistance and type 2 diabetes
}

\author{
Hung-Tsung $\mathrm{Wu}^{1,2} \cdot$ Horng-Yih $\mathrm{Ou}^{3} \cdot \mathrm{Hao}-\mathrm{Chang} \mathrm{Hung}^{3,4} \cdot \mathrm{Yu}-\mathrm{Chu} \mathrm{Su}^{4}$. \\ Feng-Hwa $\mathrm{Lu}^{2}$ • Jin-Shang $\mathrm{Wu}^{2} \cdot$ Yi-Ching Yang ${ }^{2}$ C Chao-Liang $\mathrm{Wu}^{4,5}$. \\ Chih-Jen Chang ${ }^{2,4}$
}

Received: 4 February 2016 / Accepted: 27 April 2016 / Published online: 25 May 2016

(C) Springer-Verlag Berlin Heidelberg 2016

\begin{abstract}
Aims/hypothesis Type 2 diabetes is highly correlated with nonalcoholic fatty liver disease (NAFLD). Hepatocytederived fibrinogen-related protein 1 (HFREP1) is a hepatokine that mediates NAFLD development; however, the role of HFREP1 in the development of insulin resistance and diabetes remains obscure.

Methods A total of 193 age- and sex-matched participants with normal glucose tolerance, impaired fasting glucose (IFG), impaired glucose tolerance (IGT) and newly diagnosed diabetes (NDD) were recruited for a cross-sectional study.
\end{abstract}

Hung-Tsung Wu and Horng-Yih Ou contributed equally to this work.

Electronic supplementary material The online version of this article (doi:10.1007/s00125-016-3991-7) contains peer-reviewed but unedited supplementary material, which is available to authorised users.

Chao-Liang Wu

wumolbio@mail.ncku.edu.tw

Chih-Jen Chang

changcj.ncku@gmail.com

1 Research Center of Clinical Medicine, National Cheng Kung University Hospital, College of Medicine, National Cheng Kung University, Tainan, Taiwan

2 Department of Family Medicine, National Cheng Kung University Hospital, College of Medicine, National Cheng Kung University, 138 Sheng-Li Road, Tainan 70403, Taiwan

3 Division of Endocrinology and Metabolism, Department of Internal Medicine, National Cheng Kung University Hospital, College of Medicine, National Cheng Kung University, Tainan, Taiwan

4 Institute of Basic Medical Sciences, College of Medicine, National Cheng Kung University, Tainan, Taiwan

5 Department of Biochemistry and Molecular Biology, College of Medicine, National Cheng Kung University, 1 University Road, Tainan 70101, Taiwan
Plasma HFREP1 levels were measured and multivariate linear regression analysis was used to evaluate the relationship between HFREP1, IFG, IGT and NDD. The causal relationship between HFREP1 and insulin resistance was then investigated in animal and cell models. Glucose and insulin tolerance tests, and euglycaemic-hyperinsulinaemic clamp, were used to evaluate insulin sensitivity in animals with Hfrep 1 overexpression or knockdown in liver by lentiviral vectors. HepG2 cells were used to clarify the possible mechanism of HFREP1-induced insulin resistance.

Results Plasma HFREP1 concentrations were significantly increased in participants with IFG, IGT and NDD. HFREP1 concentrations were independently associated with fasting plasma glucose levels, insulin resistance, IFG, IGT and NDD. Injection of recombinant HFREP1 or Hfrep 1 overexpression induced insulin resistance in mice, and HFREP1 disrupted insulin signalling to induce insulin resistance through an extracellular signal-regulated kinase (ERK)1/2-dependent pathway. Moreover, hepatic knockdown of HFREP1 improved insulin resistance in both mice fed a high-fat diet and $o b / o b$ mice.

Conclusions/interpretation These findings highlight the crucial role of HFREP1 in insulin resistance and diabetes, and provide a potential strategy and biomarker for developing therapeutic approaches to combat these diseases.

Keywords Biomarker · Diabetes $\cdot$ Hepatocyte-derived fibrinogen-related protein $1 \cdot$ Impaired fasting glucose . Impaired glucose tolerance

$\begin{array}{ll}\text { Abbreviations } \\ \text { ERK } & \text { Extracellular signal-regulated kinase } \\ \text { FPG } & \text { Fasting plasma glucose } \\ \text { FPI } & \text { Fasting plasma insulin }\end{array}$




\section{HFD High-fat diet}

HFREP1 Hepatocyte-derived fibrinogen-related protein 1

hsCRP High-sensitivity C-reactive protein

IFG Impaired fasting glucose

IGT Impaired glucose tolerance

IRS1 Insulin receptor substrate-1

JNK c-Jun N-terminal kinase

MCP-1 Monocyte chemoattractant protein-1

NAFLD Nonalcoholic fatty liver disease

NDD Newly diagnosed diabetes

NGT Normal glucose tolerance

\section{Introduction}

Diabetes mellitus is one of the most common noncommunicable diseases globally, and has become a major public health problem. It has been identified as an important risk factor for cardiovascular diseases and nonalcoholic fatty liver disease (NAFLD) [1]. In addition, several meta-analyses have shown that diabetes is associated with an increased risk of site-specific cancers, including cancers of the liver, colorectum and pancreas [2].

Disturbance of glucose metabolism by insulin resistance in peripheral tissues, such as the liver, skeletal muscle and adipose tissue, is one of the main mechanisms that lead to the development of type 2 diabetes [3]. When insulin resistance develops, downregulated insulin signalling increases gluconeogenic activity and contributes to increased fasting plasma glucose levels in diabetic patients $[4,5]$. Numerous studies have demonstrated that obesity causes inflammation in adipose tissue and the liver, which leads to the secretion of two types of cytokines, adipokines [6] and hepatokines [7], into the bloodstream, and this secretion then decreases insulin sensitivity and leads to the development of diabetes.

Hepatocyte-derived fibrinogen-related protein 1 (HFREP1), also called fibrinogen-like protein 1 and hepassocin, is a hepatokine that has mitogenic activity on hepatocytes [8]. Studies have revealed that HFREP1 serves as a regulator of hepatocyte growth, and is associated with the progression of hepatocellular carcinoma [9]. HFREP1 regulates hepatocyte proliferation by inducing tyrosine phosphorylation of the epidermal growth factor receptor; this leads to the transactivation of Src activity through a nonreceptormediated mechanism, and increases the phosphorylation of extracellular signal-regulated kinase (ERK) 1/2 [10]. In addition, the hepatic expression of HFREP1 is increased in mice with high-fat diet (HFD)-induced NAFLD, and this increase activates ERK1/2 to facilitate lipogenesis and leads to the development of hepatic steatosis [11]. Furthermore, HFREP1 is an acute-phase reactant that responds to inflammatory cytokines, including interleukin-6 [12]; however, the role of inflammation-induced HFREP1 expression is still obscure.

Both NAFLD and inflammation are risk factors for insulin resistance, and HFREP1 plays a crucial role in the development of NAFLD and responses to inflammation; consequently, we found increased circulating HFREP1 concentrations in patients with type 2 diabetes in a previous study [13]. However, the role of HFREP1 in insulin resistance and its levels in participants with prediabetes (impaired fasting glucose [IFG] or impaired glucose tolerance [IGT]) are unknown. In addition, the causal relationship between HFREP1 and diabetes is obscure. We thus investigated the levels of HFREP1 in participants with prediabetes, and clarified the possible mechanisms of HFREP1-induced insulin resistance in both animal and cell models.

\section{Methods}

\section{Study approval}

All patient samples were de-identified, and the participants signed an informed consent form approved by the Institutional Review Board of National Cheng Kung University Hospital (B-ER-102-418). All animal experiments were approved by the Institutional Animal Care and Use Committee of National Cheng Kung University (IACUC No: 103080).

\section{Study participants}

All participants in this case-control study were screened between June 2007 and July 2009 at National Cheng Kung University Hospital after a physical check-up. Among the study cohort, a total of 193 participants were included and classified into normal glucose tolerance (NGT), IFG, IGT and newly diagnosed diabetes (NDD) groups. The method for grouping is described in the electronic supplementary material (ESM) Methods.

Diabetes was diagnosed using a standard 75 g OGTT according to the criteria of the American Diabetes Association. None of the diabetic patients had been diagnosed as having diabetes or had been treated with insulin or any anti-diabetic agents before. The calculation of BMI, the measurement of blood pressure, and the definitions of habitual exercise, alcohol drinking and smoking habits are described in ESM Methods.

Participants with the following conditions or diseases were excluded: (1) NAFLD (diagnosed by abdominal sonography) or serum aspartate aminotransferase or alanine aminotransferase levels more than two times the normal limit; (2) testing positive for hepatitis $B$ virus surface antigen or anti-hepatitis $C$ virus antibodies, or other causes of liver disease; (3) creatinine 
$>132.6 \mu \mathrm{mol} / 1$; (4) any acute or chronic inflammatory diseases, as determined by a leucocyte count $>10,000 / \mathrm{mm}^{3}$ or clinical signs of infection; and (5) any other major diseases, including generalised inflammation or malignant diseases.

\section{Measurement of HFREP1 concentrations and metabolic variables}

After an overnight $12 \mathrm{~h}$ fast, all participants received blood tests, including fasting plasma glucose (FPG), total cholesterol, triacylglycerol, HDL-cholesterol, routine biochemistry, insulin, high-sensitivity $\mathrm{C}$-reactive protein (hsCRP) and HFREP1. Blood glucose was measured by the hexokinase method (Roche Diagnostic GmbH, Mannheim, Germany). Plasma HFREP1 concentrations (Cusabio, Wuhan, China), insulin (Mercodia AB, Uppsala, Sweden), adiponectin (AssayPro, St Charles, MO, USA), fetuin-A (Bio-vendor Laboratory Medicine, Brno, Czech Republic) and hsCRP (Immunology Consultants Laboratory, Newberg, OR, USA) were determined using commercial sandwich ELISA kits. Insulin resistance was assessed by the HOMAIR index, defined as fasting insulin $(\mathrm{pmol} / \mathrm{l}) \times$ fasting plasma glucose $(\mathrm{mmol} / \mathrm{l})] / 135$ [14]. The variables were used to identify the factors that independently associated with levels of HFREP1, and the relationship between HFREP1 and diabetes (ESM Methods).

\section{Cell culture}

Primary hepatocytes were prepared according to our previous study [11]. Human HepG2 hepatoma cells were purchased from the Bioresource Collection and Research Center (Food Industry Research and Development Institute, Hsinchu, Taiwan). Cells were maintained in Dulbecco's modified Eagle medium (Hyclone, South Logan, UT, USA) supplemented with $10 \%$ heat-inactivated fetal bovine serum.

\section{Western blot analysis}

Insulin sensitivity and related mechanisms were assessed by protein activities, including pAkt, Akt, pIR (insulin receptor), IR, pERK1/2, ERK1/2, pJNK (c-Jun N-terminal kinase), JNK, pP38, P38 (Cell Signaling Technology, Beverly, MA, USA), pIRS1 (Tyr941; Millipore, Billerica, MA, USA), HFREP1 (Proteintech Group, Wuhan, China) and actin (Millipore). See ESM Methods.

\section{Animals}

Eight-week-old C57BL/6 male mice were purchased from the Animal Center of National Cheng Kung University Medical College, and the B6.V-Lep ${ }^{\mathrm{ob}} / \mathrm{JNarl}(o b / o b)$ mice were purchased from the National Laboratory Animal Center
(Tainan, Taiwan). The animals were housed in a temperature $\left(25 \pm 1{ }^{\circ} \mathrm{C}\right)$ and humidity $(60 \pm 5 \%)$ controlled room, and kept on a 12:12 light-dark cycle (light on at 06:00 h). The mice were randomly assigned to receive different treatments. A model of type 2 diabetes in mice was produced by feeding mice with an HFD containing $34.9 \%$ fat (wt/wt; 58Y1; TestDiet, Richmond, IN, USA) or a standard chow (5001; LabDiet, St. Louis, MO, USA) for 12 weeks.

\section{Lentiviral-based transfection and delivery}

Lentiviral vectors, encoding Hfrepl, and short hairpin RNA targeted to Hfrepl, were produced as previously described [11], to overexpress or knock down HFREP1 in the liver of the mice. Control mice were injected with saline vehicle $(154 \mathrm{mmol} / \mathrm{l} \mathrm{NaCl})$. See ESM Methods.

\section{Glucose and insulin tolerance tests}

Glucose and insulin tolerance tests were performed on mice after an $8 \mathrm{~h}$ fast by intraperitoneal injection of $1.0 \mathrm{~g} / \mathrm{kg}$ glucose or $1.0 \mathrm{U} / \mathrm{kg}$ insulin, respectively. Blood samples were collected from the retro-orbital sinus of the mice at $0,30,60$, 90 and 120 min after injection of glucose or insulin for blood glucose determination using a commercial glucose assay kit (Biosystems, Barcelona, Spain), as described previously [15].

\section{In vivo insulin sensitivity in mice}

The efficacy of insulin signalling in vivo was determined as previously described [16]. Each group of mice was starved for $24 \mathrm{~h}$, and then given five units of insulin (Novo Nordisk, Bagsvaerd, Denmark) in the inferior vena cava. After 5 min, the liver and hind leg muscles were removed for western blot analysis.

\section{Hyperinsulinaemic-euglycaemic clamp}

Mice were studied in an unrestrained state using the euglycaemic-hyperinsulinaemic clamp technique as previously described [17]. Data for the calculation of total body glucose uptake and endogenous glucose production were determined as previously described [18]. See ESM Methods.

\section{Statistics}

All clinical data were analysed using nonparametric statistics (Kruskal-Wallis test with post-hoc tests) and presented as means $\pm \mathrm{SD}$, or median, upper and lower quartiles. In vitro experiments were analysed using parametric statistics (ANOVA test with Bonferroni-corrected two-tailed Student's $t$ tests as post-hoc tests) and presented as mean \pm SEM. All of 
the statistical analyses were conducted with SPSS software (version 17.0; SPSS, Chicago, IL, USA). Significance was set at $p<0.05$.

\section{Results}

\section{Human study}

Plasma HFREP1 concentrations were increased in prediabetes and diabetes, and fasting blood glucose and HOMA-IR were independently associated with HFREP1 levels The anthropometric features of each group studied along with the results of routine biochemical tests were measured. There were significant differences in FPG $(p<0.001)$, hsCRP $(p<0.001)$, triacylglycerol $(p<0.05)$, HDL-cholesterol $(p<0.01)$, adiponectin $(p<0.05)$, fetuin-A $(p<0.05)$ and HOMA-IR $(p<0.01)$ among participants with NGT, IFG, IGT and NDD (Table 1). Plasma HFREP1 concentrations were significantly increased in IFG $(p<0.001)$, IGT $(p=0.009)$ and NDD $(p<0.001)$ groups compared with the NGT group (Fig. 1).

To further determine the independent factors associated with plasma HFREP1 concentrations, we performed

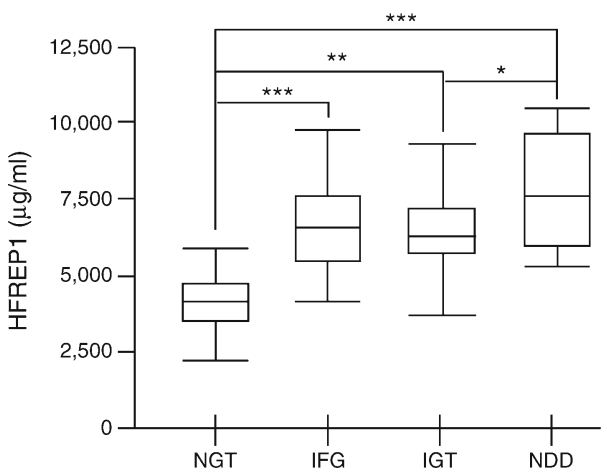

Fig. 1 Plasma HFREP1 concentrations were increased in IFG, IGT and NDD groups. Box and whisker plot of plasma HFREP1 concentrations in participants with NGT $(n=49)$, IFG $(n=46)$, IGT $(n=49)$ and NDD $(n=49)$. The line inside the box represents the median of the distribution, the box top and bottom values are defined by the 25th and 75th percentiles, and the whiskers are the minimum and maximum values. ${ }^{*} p<0.05,{ }^{* *} p<0.01$ and ${ }^{* * *} p<0.001$ compared with the indicated groups or control group

multivariate linear regression analysis. Both stepwise and backward selection strategies showed a consistent model, which is summarized in Table 2. By use of NGT as a reference group, we found that FPG $(\beta=344.49 ; p<0.001)$ was independently associated with HFREP1 concentrations after adjustment for the confounding factors listed in Model 1 of
Table 1 Clinical characteristics of the study participants in each group

\begin{tabular}{|c|c|c|c|c|c|}
\hline Characteristic & NGT & IFG & IGT & NDD & $p$ \\
\hline$n$ & 49 & 46 & 49 & 49 & - \\
\hline Age (years) & $61.0 \pm 11.6$ & $61.3 \pm 12.4$ & $62.5 \pm 12.0$ & $61.8 \pm 11.7$ & NS \\
\hline $\operatorname{Sex}(\mathrm{F} / \mathrm{M})$ & $20 / 29$ & $18 / 28$ & $20 / 29$ & $20 / 29$ & NS \\
\hline BMI $\left(\mathrm{kg} / \mathrm{m}^{2}\right)$ & $22.8 \pm 2.7$ & $24.0 \pm 2.5$ & $23.9 \pm 3.2$ & $23.7 \pm 3.0$ & NS \\
\hline $\mathrm{SBP}(\mathrm{mmHg})$ & $123.6 \pm 17.5$ & $128.3 \pm 19.4$ & $129.3 \pm 17.3$ & $127.8 \pm 18.8$ & NS \\
\hline DBP (mmHg) & $72.0 \pm 9.6$ & $74.0 \pm 9.2$ & $74.2 \pm 10.8$ & $74.8 \pm 10.5$ & NS \\
\hline FPG (mmol/1) & $4.8 \pm 0.4$ & $5.8 \pm 0.3$ & $4.9 \pm 0.6$ & $7.4 \pm 3.4$ & $<0.001$ \\
\hline $\operatorname{ALT}(\mathrm{U} / \mathrm{l})$ & $23.3 \pm 9.4$ & $25.8 \pm 18.7$ & $21.6 \pm 8.8$ & $29.1 \pm 21.9$ & NS \\
\hline AST (U/l) & $25.8 \pm 6.4$ & $24.9 \pm 8.5$ & $23.6 \pm 4.9$ & $27.2 \pm 13.5$ & NS \\
\hline Creatinine $(\mu \mathrm{mol} / \mathrm{l})$ & $75.3 \pm 15.3$ & $77.0 \pm 17.6$ & $79.8 \pm 16.8$ & $75.2 \pm 21.2$ & NS \\
\hline hsCRP (nmol/l) & $26.8 \pm 42.1$ & $27.0 \pm 41.7$ & $24.8 \pm 41.3$ & $58.2 \pm 83.7$ & $<0.001$ \\
\hline Total cholesterol $(\mathrm{mmol} / \mathrm{l})$ & $5.30 \pm 1.01$ & $5.30 \pm 1.00$ & $5.29 \pm 0.98$ & $5.39 \pm 1.23$ & NS \\
\hline Triacylglycerol $(\mathrm{mmol} / \mathrm{l})^{\mathrm{a}}$ & $1.11 \pm 0.43$ & $1.30 \pm 0.76$ & $1.30 \pm 0.74$ & $1.64 \pm 1.24$ & $<0.05$ \\
\hline HDL-cholesterol (mmol/l) & $1.56 \pm 0.43$ & $1.43 \pm 0.39$ & $1.36 \pm 0.39$ & $1.29 \pm 0.29$ & $<0.01$ \\
\hline LDL-cholesterol (mmol/l) & $3.23 \pm 0.97$ & $3.28 \pm 0.90$ & $3.33 \pm 0.89$ & $3.34 \pm 0.98$ & NS \\
\hline Adiponectin $(\mu \mathrm{mol} / \mathrm{dl})$ & $4.77 \pm 4.01$ & $4.51 \pm 3.15$ & $4.21 \pm 4.30$ & $3.45 \pm 1.95$ & $<0.05$ \\
\hline Fetuin-A $(\mu \mathrm{mol} / 1)$ & $5.08 \pm 1.02$ & $5.21 \pm 1.70$ & $5.46 \pm 1.46$ & $5.55 \pm 1.33$ & $<0.05$ \\
\hline HOMA-IR & $0.52 \pm 0.52$ & $0.85 \pm 0.55$ & $0.59 \pm 0.48$ & $0.92 \pm 0.88$ & $<0.01$ \\
\hline Smoking (\%) & 4.08 & 6.52 & 4.08 & 6.12 & NS \\
\hline Habitual exercise (\%) & 8.16 & 6.52 & 2.04 & 2.04 & NS \\
\hline
\end{tabular}

Data expressed as means $\pm \mathrm{SD}$ or percentage

${ }^{\text {a }}$ Kruskal-Wallis test

ALT, alanine aminotransferase; AST, aspartate aminotransferase; DBP, diastolic blood pressure; SBP, systolic blood pressure 
Table 2 Regression analysis between plasma HFREP1 concentrations and clinical variables

\begin{tabular}{|c|c|c|c|c|c|c|}
\hline \multirow[t]{2}{*}{ Variable } & \multicolumn{2}{|l|}{ Model 1} & \multicolumn{2}{|l|}{ Model 2} & \multicolumn{2}{|l|}{ Model 3} \\
\hline & $\beta(95 \% \mathrm{CI})$ & $p$ & $\beta(95 \% \mathrm{CI})$ & $p$ & $\beta(95 \% \mathrm{CI})$ & $p$ \\
\hline Age & $21.81(-7.29,50.91)$ & NS & $9.21(-21.17,39.58)$ & NS & $20.12(-7.29,47.52)$ & NS \\
\hline Sex & $-127.37(-944.50,689.76)$ & NS & $-11.70(-875.85,852.46)$ & NS & $77.73(-703.32,858.78)$ & NS \\
\hline BMI & $59.81(-52.02,171.63)$ & NS & $34.03(-84.20,152.26)$ & NS & $77.42(-33.77,178.61)$ & NS \\
\hline FPG & $344.49(191.86,497.12)$ & $<0.001$ & - & - & - & - \\
\hline FPI & $28.57(-37.38,94.52)$ & NS & - & - & - & - \\
\hline HOMA-IR & - & - & $617.84(117.33,1118.36)$ & 0.016 & - & - \\
\hline IFG vs NGT & - & - & - & - & $1831.46(1068.16,2594.75)$ & $<0.001$ \\
\hline IGT vs NGT & - & - & - & - & $1395.95(635.40,2156.50)$ & $<0.001$ \\
\hline NDD vs NGT & - & - & - & - & $2426.52(1648.94,3204.10)$ & $<0.001$ \\
\hline Adiponectin & $25.57(-37.38,94.52)$ & NS & $24.10(-64.07,112.28)$ & NS & $42.34(-37.70,122.38)$ & NS \\
\hline Fetuin-A & $35.52(-167.56,238.61)$ & NS & $5.60(-207.89,219.09)$ & NS & $83.82(-111.30,278.94)$ & NS \\
\hline hsCRP & $1.06(-4.22,6.34)$ & NS & $2.73(-2.71,8.17)$ & NS & $1.116(-3.99,6.22)$ & NS \\
\hline SBP & $-13.23(-31.14,4.67)$ & NS & $-6.91(-25.49,11.68)$ & NS & $-14.37(-31.31,2.56)$ & NS \\
\hline ALT & $-7.32(-25.23,10.58)$ & NS & $-6.45(-25.18,12.28)$ & NS & $-8.14(-25.25,8.96)$ & NS \\
\hline Creatinine & $-2.92(-24.38,18.54)$ & NS & $-5.04(-27.72,17.64)$ & NS & $-2.95(-23.46,17.57)$ & NS \\
\hline $\log _{10}$ triacylglycerol & $156.47(-1470.82,1785.75)$ & NS & $1122.84(-496.10,2741.77)$ & NS & $1156.65(-295.42,1929.39)$ & NS \\
\hline HDL-cholesterol & $230.88(-670.05,1131.80)$ & NS & $485.07(-465.91,1436.05)$ & NS & $820.16(-55.36,1695.67)$ & NS \\
\hline Smoking & $122.53(-1041.45,1286.71)$ & NS & $291.49(-926.66,1509.64)$ & NS & $62.18(-1036.34,1929.39)$ & NS \\
\hline Habitual exercise & $973.12(-351.34,2297.57)$ & NS & $665.66(-712.05,2043.36)$ & NS & $669.42(-590.55,2151.58)$ & NS \\
\hline
\end{tabular}

Dependent variable: plasma HFREP1 concentrations

ALT, alanine aminotransferase; SBP, systolic blood pressure

Table 1. When substituting FPG and fasting plasma insulin (FPI) with HOMA-IR in Model 2, we found that HOMA-IR $(\beta=617.84 ; p=0.016)$ was independently associated with HFREP1 concentrations. Finally, to investigate the independent effect of glycaemic status on circulating HFREP1 concentrations, we used NGT as a reference group and found that IFG $(\beta=1831.46 ; p<0.001)$, IGT $(\beta=1395.95$; $p<0.001)$ and NDD $(\beta=2426.52 ; p<0.001)$ were all independently associated with HFREP1 concentrations (Model 3).

\section{Animal and cell studies}

\section{Administration of HFREP1 recombinant protein induced} insulin resistance in mice In order to confirm the relationship between HFREP1 and diabetes, a diabetic animal model was used. Corrections of hyperglycaemia in HFD-induced type 2 diabetic mice by glucose-lowering agents (Fig. 2a), including metformin or rosiglitazone, decreased the hepatic expression of HFREP1 and the concentration of HFREP1 in the circulation (Fig. 2b,c).

Although we had found significant correlations between hyperglycaemia/diabetes and HFREP1 in human and animal models, the causal relationship between hyperglycaemia/ diabetes and HFREP1 remained obscure. In view of this, we injected HFREP1 recombinant protein into wild-type mice to evaluate the effects of HFREP1 on the development of insulin resistance and diabetes (Fig. 3). After a daily injection of HFREP1 for 7 days, the body weight of the mice in the HFREP1-treated group was not affected as compared with those in the saline-treated control group (Fig. 3a). However, FPG (Fig. 3b), insulin levels (Fig. 3c) and HOMA-IR (Fig. 3d) were significantly increased after the HFREP1 treatment, indicating the development of insulin resistance. To confirm this, both glucose and insulin tolerance tests (Fig. 3e,f) as well as hyperinsulinaemic-euglycaemic clamps (Fig. 3g-j) were used. The results revealed that HFREP1 induced impaired glucose utility and insulin resistance. Furthermore, we found that HFREP1 impaired insulin signalling in both the liver (Fig. 3k,1) and skeletal muscle (Fig. 3m,n) in mice.

Hepatic Hfrep1 overexpression by lentiviral transfection induced insulin resistance in mice Since the effects of endotoxin contamination in HFREP1 recombinant protein could not be excluded in the HFREP1-induced insulin resistance in mice, we used a lentiviral vector containing Hfrepl to overexpress hepatic Hfrepl in mice (Fig. 4). As shown in Fig. 4, hepatic Hfrepl overexpression was achieved by a portal vein injection of a lentiviral vector containing 
a

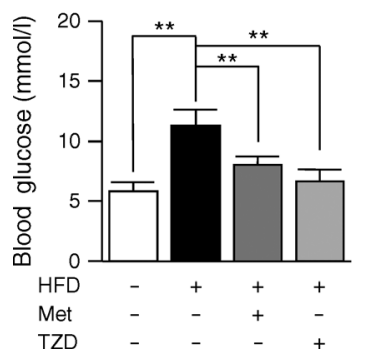

b

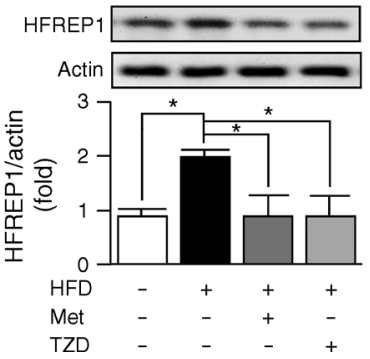

C

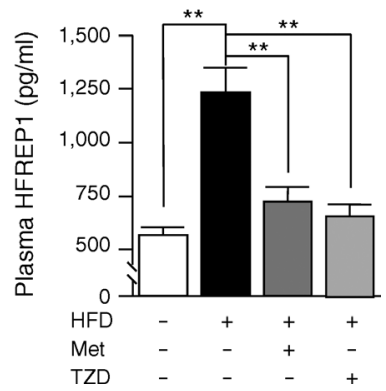

Fig. 2 Correction of hyperglycaemia in mice with HFD-induced type 2 diabetes decreased levels of HFREP1. Fasting blood glucose levels (a), hepatic HFREP1 expression (b) and plasma HFREP1 concentrations (c) in mice with HFD-induced type 2 diabetes that were treated with

Hfrep1 (Fig. 4a), and the circulating HFREP1 concentrations were significantly increased (Fig. 4b), whereas the lentiviral vector transfections did not affect the body weight of each group (Fig. 4c). In parallel with the results for the HFREP1 recombinant protein in the development of insulin resistance (Fig. 3), hepatic overexpression of Hfrepl in mice also increased the levels of fasting plasma glucose (Fig. 4d) and insulin (Fig. 4e), as well as the HOMA-IR index (Fig. 4f). metformin $(5 \mathrm{~g} / \mathrm{kg}$ diet) or rosiglitazone $(50 \mathrm{mg} / \mathrm{kg}$ diet $)$ for 2 weeks. ${ }^{*} p<0.05$ and ${ }^{* *} p<0.01$ compared with the indicated groups or control group ( $n=8-10$ for each group). Met, metformin; TZD, thiazolidedione (rosiglitazone)

Further experiments, including glucose and insulin tolerance tests (Fig. 4g,h), as well as the use of hyperinsulinaemiceuglycaemic clamps (Fig. 4i-1), indicated that hepatic Hfrepl overexpression by lentiviral vector induced impaired glucose utility and insulin resistance. Moreover, impaired insulin signals in both liver (Fig. $4 \mathrm{~m}, \mathrm{n}$ ) and skeletal muscle (Fig. 4o,p) were observed in the Hfrep1-overexpressed group, as compared with the control group. a

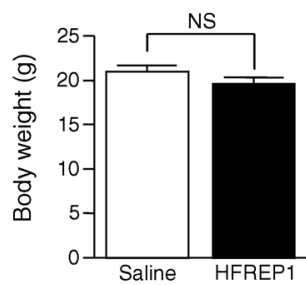

e

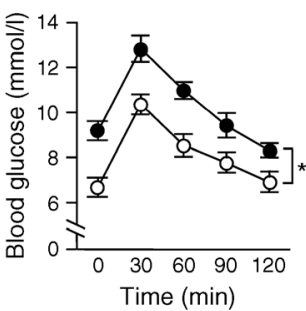

j $\quad k$

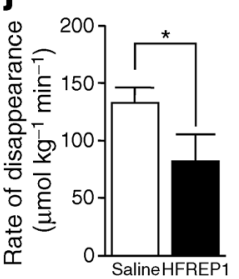

k b

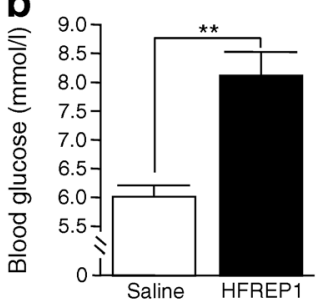

C

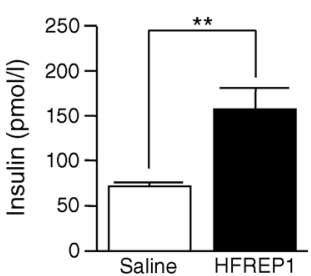

$\mathbf{h}_{-}$

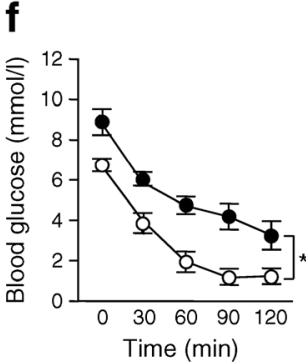

$\mathbf{g}_{-}$

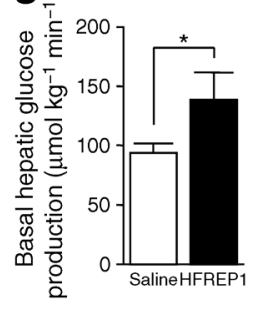

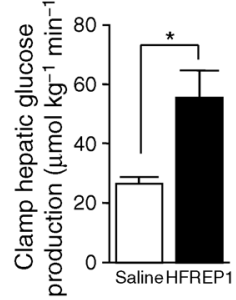

d

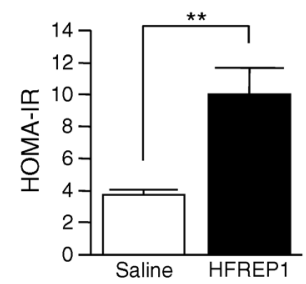

i

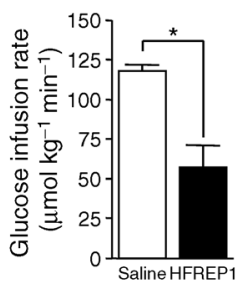

m

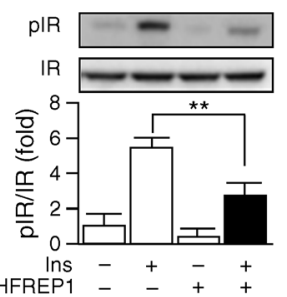

n

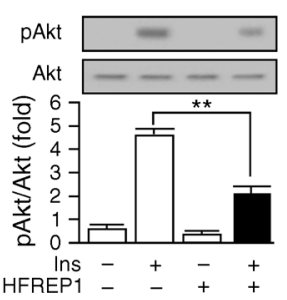

Fig. 3 Intraperitoneal injection of HFREP1 induced insulin resistance in mice. Eight-week-old mice were intraperitoneally injected with $1 \mu \mathrm{g} / \mathrm{kg}$ HFREP1 recombinant protein for 7 days, and the body weight (a), fasting blood glucose (b) and fasting blood insulin (c) of each group were measured, and HOMA-IR was calculated (d). In vivo insulin sensitivity was determined by a glucose tolerance test (e), insulin tolerance test (f) and hyperinsulinaemic-euglycaemic clamp $(\mathbf{g}-\mathbf{j})$, and the insulin downstream signals were also obtained, such as pIR and pAkt in both liver $(\mathbf{k}, \mathbf{l})$ and skeletal muscle $(\mathbf{m}, \mathbf{n})$. White circles, saline-treated group; black circles, HFREP1 recombinant protein-injected group; ${ }^{*} p<0.05$, and $* * p<0.01$ compared with the indicated groups $(n=8$ for each group). Ins, insulin 
a

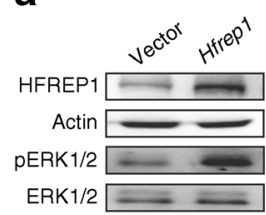

b

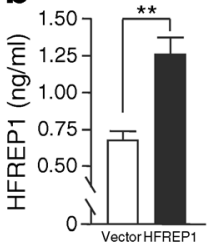

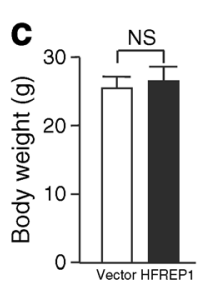

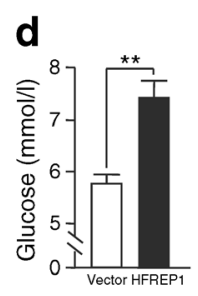

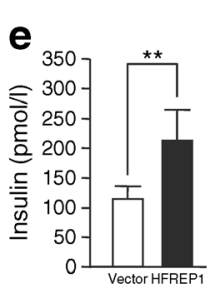

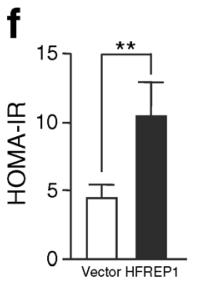

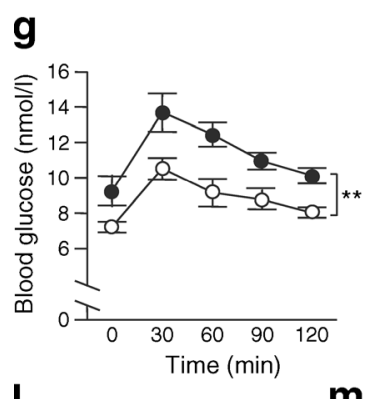

I

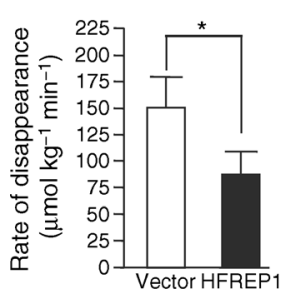

m

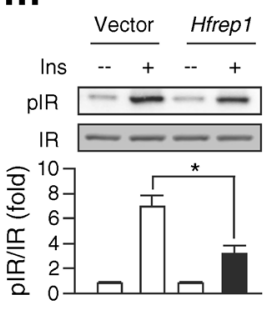

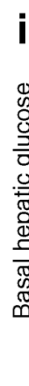

i

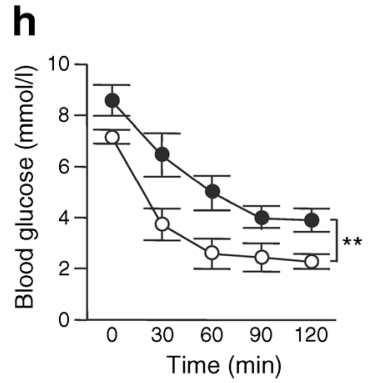

n

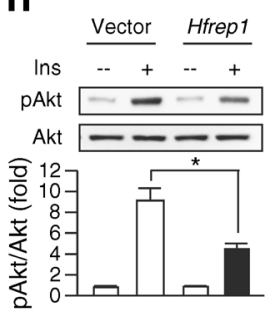

$\mathbf{0}$

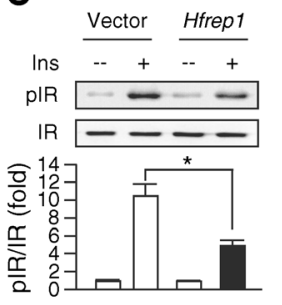

k

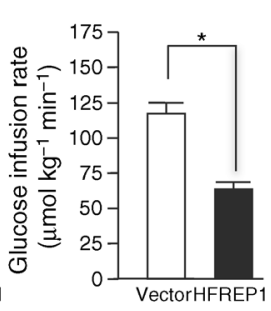

Fig. 4 Hepatic Hfrepl overexpression induced insulin resistance in mice. Eight-week-old mice were injected with a lentiviral vector containing Hfrep1 through the portal vein. The overexpression of hepatic Hfrep1 (a), and the elevated concentrations of plasma HFREP1 were confirmed after 2 weeks of the injection (b). Body weight (c), fasting blood glucose (d) and fasting blood insulin (e) of each group were measured, and HOMA-IR was also calculated (f). In vivo insulin sensitivity was

HFREP1 induces insulin resistance through an ERK1/2dependent pathway in hepatocytes In order to investigate the detailed mechanisms of HFREP1-induced insulin resistance, we used HepG2 cells, a reliable cell model for metabolic research (Fig. 5). HFREP1 treatment in HepG2 cells significantly disrupted insulin signalling, including the phosphorylation of insulin receptor (Fig. 5a), insulin receptor substrate-1 (Fig. 5b) and Akt (Fig. 5c) in a dose-dependent manner (Fig. 5d). In addition, HFREP1 increased the phosphorylation of mitogen-activated protein kinases (MAPKs) (Fig. 5e), and blockade of ERK1/2 by U0126 (an ERK1/2 inhibitor) reversed HFREP1-induced insulin resistance in a dose-dependent manner (Fig. 5f). By contrast, pretreatment with JNK1/2 (Fig. $5 \mathrm{~g}$ ) or P38 (Fig. 5h) inhibitor showed no significant effects, indicating that HFREP1 induces insulin resistance through an ERK1/2-dependent pathway. In parallel with the results in HepG2 cells, HFREP1 also blocked insulin signalling through an ERK1/2-dependent pathway in primary hepatocytes (Fig. 5i).

Knockdown of Hfrep1 improved HFD-induced insulin resistance in mice In view of the finding that HFREP1 determined by a glucose tolerance test (g), insulin tolerance test (h) and hyperinsulinaemic-euglycaemic clamp (i-l), and the insulin downstream signals were also obtained, such as pIR and pAkt in both liver $(\mathbf{m}, \mathbf{n})$ and skeletal muscle $(\mathbf{o}, \mathbf{p})$. White circles, lentiviral vector-injected control group; black circles, lentiviral vector with Hfrepl-injected group; ${ }^{*} p<0.05$ and ${ }^{*} p<00.01$ compared with the indicated groups $(n=8$ for each group). Ins, insulin

contributes to the development of insulin resistance, we further evaluated whether knockdown of HFREP1 improved insulin resistance in mice fed an HFD (Fig. 6). Knockdown of hepatic HFREP1 by lentiviral vector containing short hairpin RNA targeted to Hfrepl was confirmed by western blots (Fig. 6a), and the circulating HFREP1 concentrations were significantly decreased after hepatic HFREP 1 knockdown (Fig. 6b). Gene transfection by lentiviral vector showed no significant effects on HFD-induced weight gain (Fig. 6c). However, blood glucose (Fig. 6d) and insulin levels (Fig. 6e), as well as HOMA-IR (Fig. 6f), were significantly decreased in HFREP1 knockdown HFD mice, as compared with the lentiviral-vector-delivered control group. Further examinations of the improved insulin sensitivity in HFREP1 knockdown HFD mice were also carried out with glucose (Fig. 6g) and insulin tolerance tests (Fig. 6h) as well as hyperinsulinaemic-euglycaemic clamps (Fig. 6i-1). The results indicated that knockdown of HFREP1 in HFD mice significantly increased glucose utility and insulin sensitivity, and improved HFD-impaired insulin signalling in both the liver (Fig. $6 \mathrm{~m}, \mathrm{n}$ ) and skeletal muscle (Fig. 6o,p). 
a

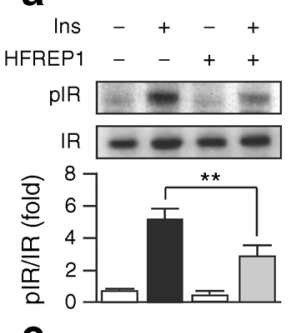

C

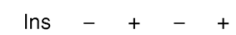

HFREP1 $-\quad-\quad+\quad+$

pAkt -

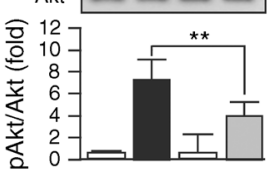

$\mathbf{f}$
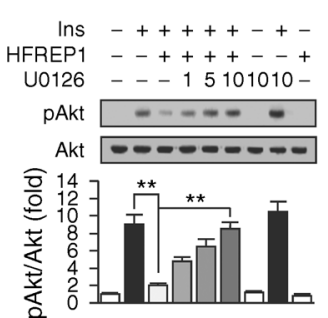

b

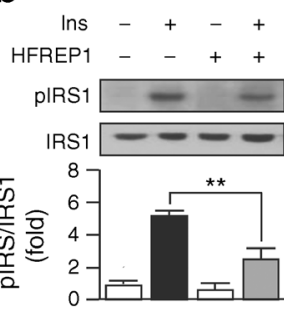

d

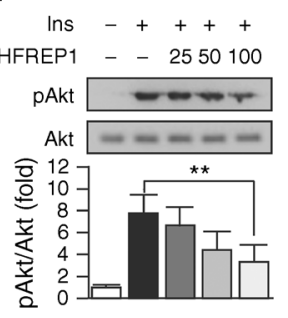

e
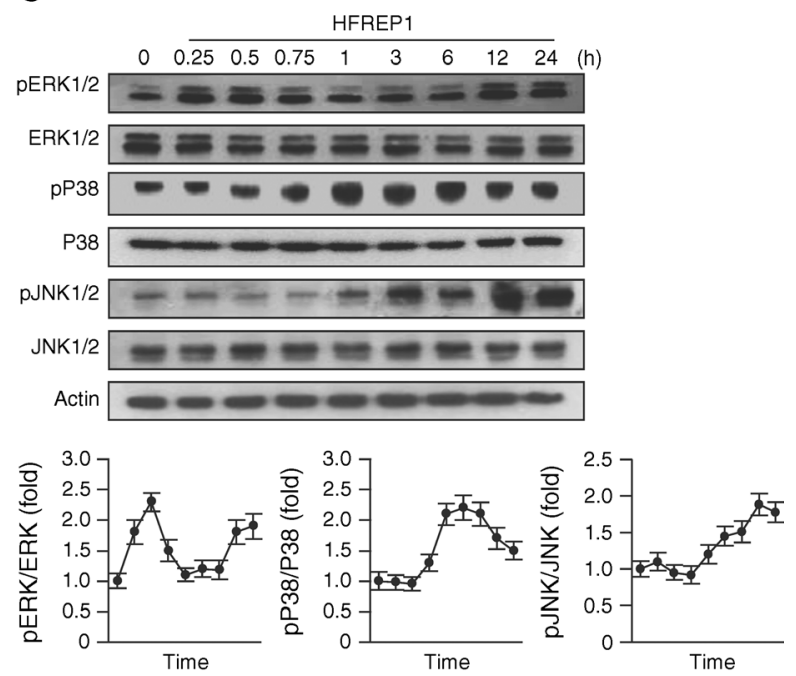

\section{g}

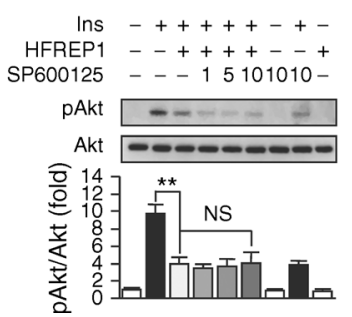

h

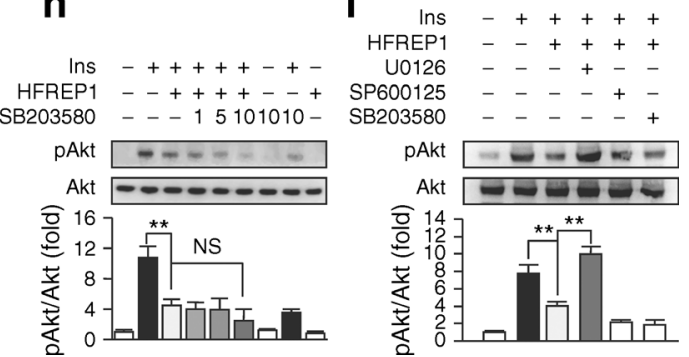

P38 and JNK1/2 (e). HepG2 cells (f-h) or primary hepatocytes (i) were pre-treated with $10 \mu \mathrm{mol} / 1$ or different doses of ERK1/2 inhibitor (U0126) (f), JNK1/2 inhibitor (SP600125) (g, i), or P38 inhibitor (SB203580) (h, i) for $30 \mathrm{~min}$, and then treated with $1 \mu \mathrm{mol} / \mathrm{l}$ insulin for 30 min for the detection of Akt phosphorylation. The data was obtained from at least three individual experiments. ${ }^{* *} p<0.01$ compared with the indicated groups. Ins, insulin

\section{Knockdown of Hfrep1 improved insulin resistance in} $\boldsymbol{o b} / \boldsymbol{o b}$ mice In addition to the evaluation of hepatic HFREP1 knockdown on insulin sensitivity in HFD mice, we also investigated the effects of hepatic HFREP1 knockdown in genetic diabetic ob/ob mice. As shown in Fig. 7a, hepatic Hfrep1 expression and plasma HFREP1 concentrations were increased in $o b / o b$ mice with increased ERK1/2 activity. After hepatic knockdown of HFREP1, plasma HFREP1 levels were significantly decreased (Fig. 7b), whereas the body weight of the mice was not changed (Fig. 7c). Following the knockdown of HFREP1 levels in $o b / o b$ mice, fasting blood glucose (Fig. 7d) and insulin levels (Fig. 7e), as well as HOMA-IR (Fig. 7f), were significantly decreased. In order to confirm the improvement of insulin resistance in $o b / o b$ mice after HFREP1 knockdown, glucose tolerance tests (Fig. 7g), insulin tolerance tests (Fig. 7h) and clamp studies were used (Fig. 7i-1). We found that HFREP1 knockdown improved insulin resistance, and improved the impairment of insulin signalling in both the liver (Fig. $7 \mathrm{~m}, \mathrm{n}$ ) and skeletal muscle (Fig. 7o,p) in $o b / o b$ mice.

\section{Discussion}

To the best of our knowledge, this study is the first to clarify the mechanisms of HFREP1 in the development of insulin resistance in diabetes. In the human study, we demonstrated a significant association between HFREP1 and FPG, insulin resistance and diabetes. However, the cross-sectional design does not allow for causal inferences. In view of this fact, we investigated the detailed mechanisms in animal and cell models.

Many previous studies have shown that both NAFLD and type 2 diabetes are closely related to insulin resistance in their pathogenesis [19]. The presence of NAFLD increases the risk of future diabetes independent of BMI, body fat and measures of insulin sensitivity in nondiabetic individuals [20]. Accumulating evidence also suggests that hepatokines produced by the liver affect insulin signalling in insulin-responsive tissues. These humoral factors include retinol binding protein 4, fibroblast growth factor 21 and fetuin-A 
a

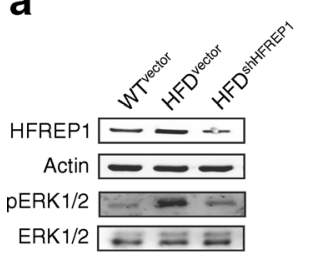

g

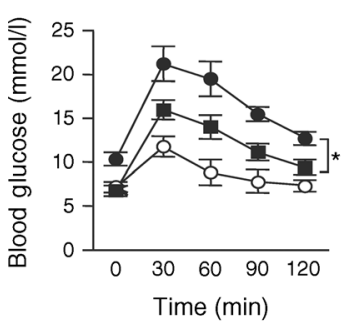

b

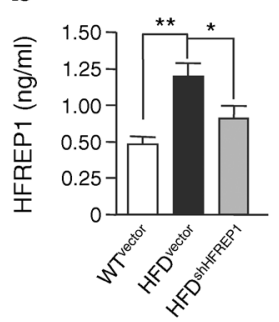

h

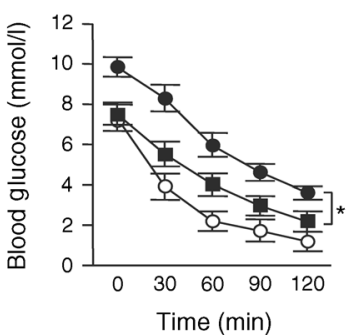

C d

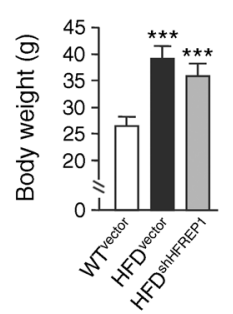

i

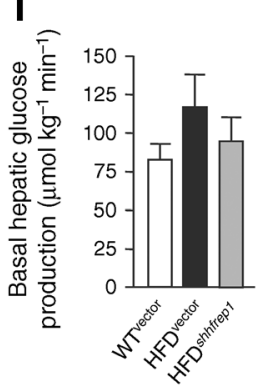

e
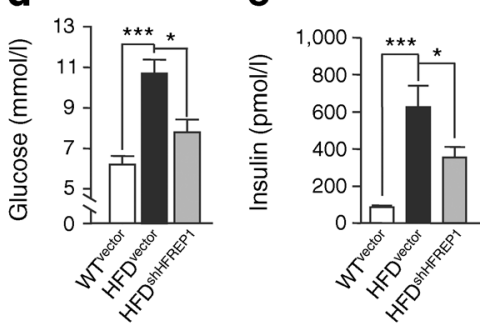

j

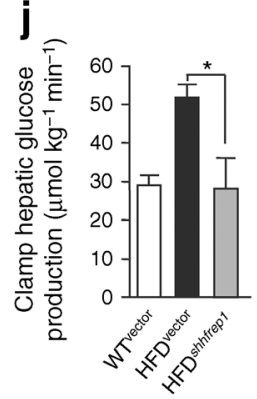

f

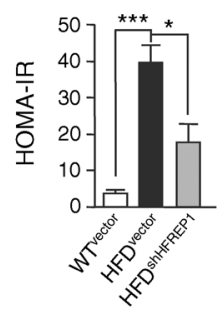

$\mathbf{k}$

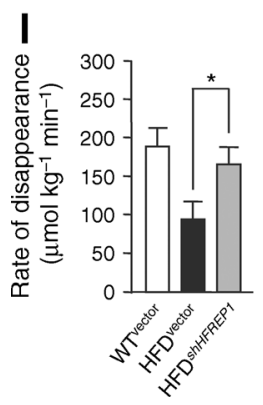

m n

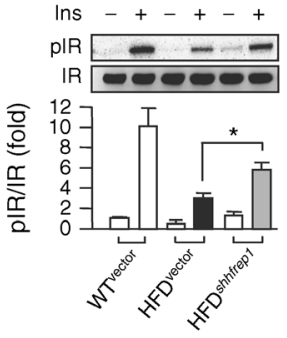

O

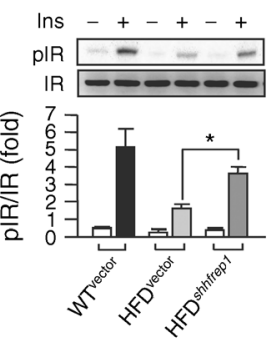

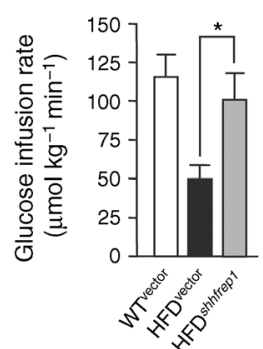

p

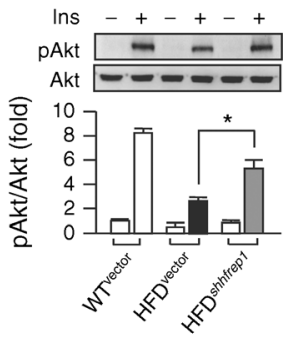

Fig. 6 Knockdown of hepatic HFREP1 improved HFD-induced insulin resistance. Eight-week-old mice were fed an HFD for 12 weeks. The mice were injected with a lentiviral vector containing short hairpin RNA targeted to Hfrepl (HFD ${ }^{\text {shHfrepl }}$ ) through the portal vein. The knockdown of hepatic HFREP1 (a) and reduced plasma HFREP1 concentrations were confirmed 2 weeks after the injection (b). The body weight (c), fasting blood glucose (d) and fasting blood insulin (e) of each group were measured, and HOMA-IR was also calculated (f). In vivo insulin sensitivity was determined by a glucose tolerance test (g), insulin tolerance test (h) and hyperinsulinaemic-euglycaemic clamp (i-l), and the insulin downstream signals were also obtained, such as pIR and pAkt in both liver $(\mathbf{m}, \mathbf{n})$ and skeletal muscle $(\mathbf{o}, \mathbf{p})$. White circles, lentiviral vector-injected chow-fed wild-type control group; black circles, lentiviral vector-injected HFD-fed group; black squares, lentiviral vector with shHfrep 1 -injected HFD-fed group; ${ }^{*} p<0.05,{ }^{* *} p<0.01$ and $* * * p<0.001$ compared with the indicated groups ( $n=8$ for each group). Ins, insulin

contribute to the development of insulin resistance [23]. Levels of several inflammatory factors that play critical roles in insulin resistance and diabetes, such as monocyte chemoattractant protein-1 (MCP-1), are increased in patients with diabetes. An increase in MCP-1 levels activates ERK1/2 to decrease glucose utility in muscle cells [24]. In addition, the activity of ERK is increased in the livers of mice with diet-induced and genetic obesity [23]. Consistent with this previous study, we also observed elevated ERK activities in both HFD-induced and $o b / o b$ diabetic mice. Also, we found that HFREP1 induced insulin resistance via an ERK1/2-dependent mechanism in hepatocytes. Consistent with previous studies, we found that the elevated HFREP1 levels in the circulation might activate ERK1/2 to disrupt insulin signalling in peripheral tissues and contribute to the development of type 2 diabetes. 
a

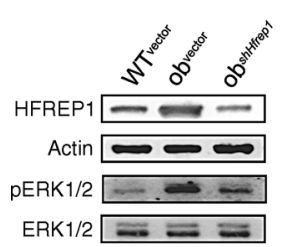

g

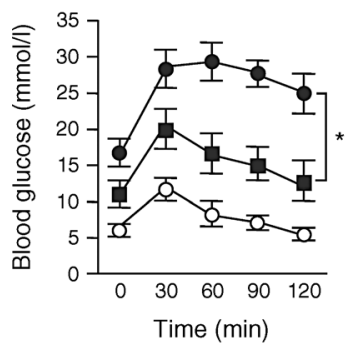

I

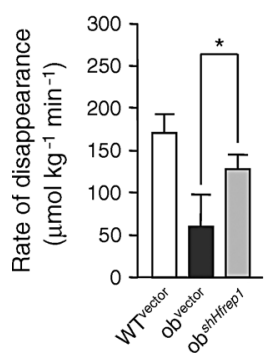

m

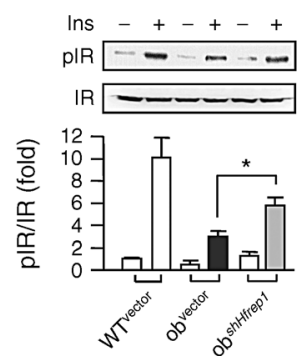

n

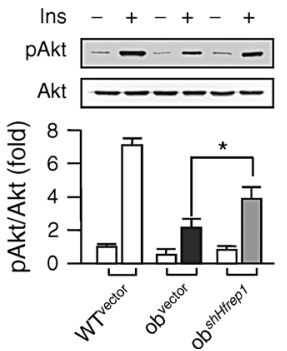

d

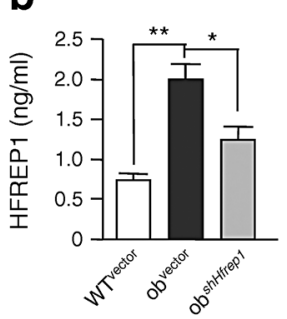

h

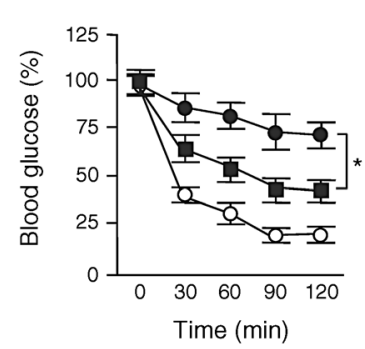

C

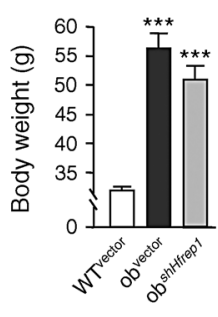

i

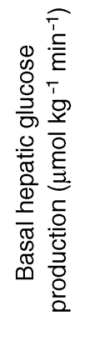

e
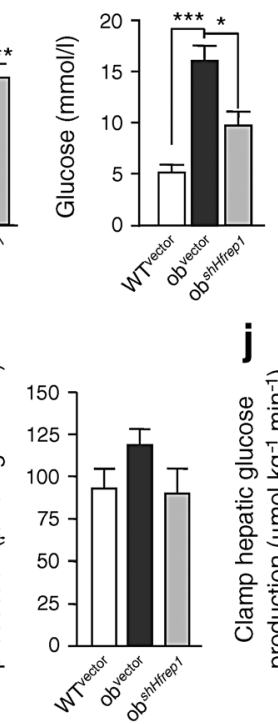

0

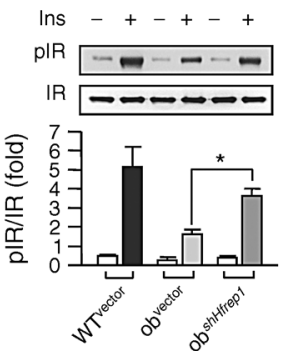

f

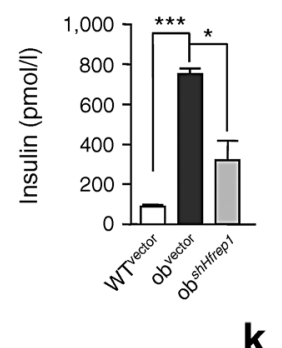

k
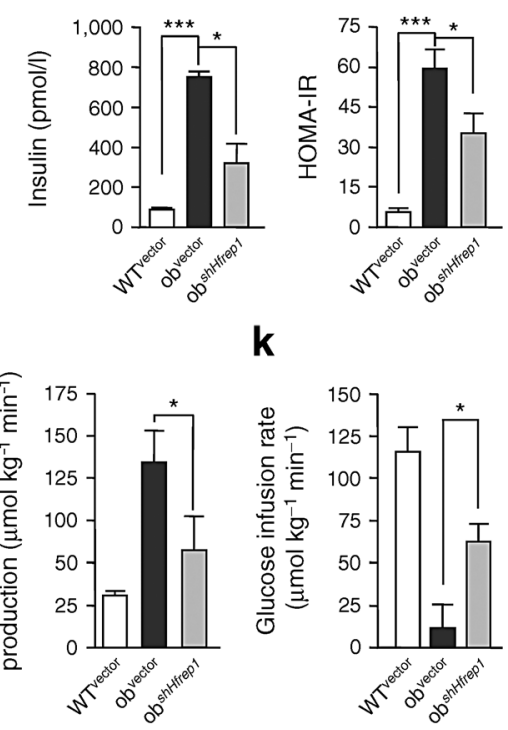

p

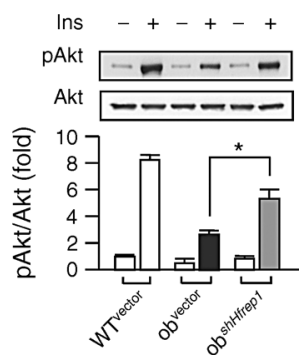

Fig. 7 Knockdown of hepatic HFREP1 improved insulin resistance in $o b / o b$ mice. Twelve-week-old $o b / o b$ mice were injected with a lentiviral vector containing short hairpin RNA targeted to Hfrepl ( $\mathrm{ob}^{\text {shHfrepl }}$ ) through the portal vein (a). The knockdown of hepatic HFREP1 and plasma HFREP1 concentrations were confirmed 2 weeks after the injection (b). The body weight (c), fasting blood glucose (d) and fasting blood insulin (e) of each group were measured, and HOMA-IR was also calculated (f). In vivo insulin sensitivity was determined by a glucose tolerance test (g), insulin tolerance test (h) and hyperinsulinaemiceuglycaemic clamp (i-l), and the insulin downstream signals were also obtained, such as pIR and pAkt in both liver $(\mathbf{m}, \mathbf{n})$ and skeletal muscle $(\mathbf{o}, \mathbf{p})$. White circles, lentiviral vector-injected chow-fed wild-type control group; black circles, lentiviral vector-injected $o b / o b$ mice group; black squares, lentiviral vector with shHfrepl-injected ob/ob mice group; $*_{p}<0.05, * * p<0.01$ and $* * * p<0.001$ compared with the indicated groups ( $n=6-8$ for each group). Ins, insulin
It has recently been reported that HFREP1 is expressed in adipose tissue and that HFREP1 regulates lipid metabolism [25], implying that HFREP1 might have a potential role in the distribution and function of adipose tissue. In the present study, there were no direct effects of hepatic HFREP1 delivery on the adipose tissue, since portal vein injection of lentiviral vectors is a route specific for hepatic gene delivery [26]. In addition, we found no significant correlations between plasma HFREP1 concentrations, BMI, lipid profile, inflammation (hsCRP) and adipokine (adiponectin), as shown in Table 2. However, HFREP1 concentrations showed significant correlations with insulin resistance. Thus, we focused on the role of HFREP1 in the development of insulin resistance.

Taken together, we found that HFREP1 plays a crucial role in the development of insulin resistance by regulating ERK1/2 activity. Elevated HFREP1 levels might increase ERK1/2 activity, block insulin signalling and induce insulin resistance. Our results suggest that increased HFREP1 levels are a risk factor for both insulin resistance and diabetes, and that HFREP1 might be a potential biomarker for the diagnosis of prediabetes.

Acknowledgements We thank H.-S. Liu and S.-H. Lan (Department of Microbiology and Immunology, National Cheng Kung University) for discussions about the article, and P. Wu (Institute for Science \& Technology in Medicine, Keele University, Keele, UK), L.-S. Chang and L.-F. Chang (Division of Endocrinology and Metabolism, Department of Internal Medicine, National Cheng Kung University Hospital) for technical assistance.

Funding The work was supported by grants from the Ministry of Science and Technology of the Republic of China (103-2314-B006-027-MY2 and 103-2314-B-006-065) and National Cheng Kung University Hospital (NCKUH-10306023). 
Duality of interest The authors declare that there is no duality of interest associated with this manuscript.

Contribution statement $\mathrm{H}-\mathrm{TW}, \mathrm{H}-\mathrm{YO}$ and C-JC were responsible for designing and conducting the study, interpreting the data and writing the manuscript. Y-CS and C-LW contributed to data collection, analysis and interpretation, as well as the writing of the manuscript concerning the animal experiments. F-HL, H-CH, J-SW and Y-CY contributed to data interpretation, analysis and the writing of the manuscript concerning the clinical study. All of the authors approved the final version of the manuscript, and $\mathrm{C}-\mathrm{JC}$ is the guarantor of this work.

\section{References}

1. Noto H, Goto A, Tsujimoto T, Osame K, Noda M (2013) Latest insights into the risk of cancer in diabetes. J Diabetes Investig 4: 225-232

2. El-Serag HB, Hampel H, Javadi F (2006) The association between diabetes and hepatocellular carcinoma: a systematic review of epidemiologic evidence. Clin Gastroenterol Hepatol 4:369-380

3. Pirola L, Johnston AM, Van Obberghen E (2004) Modulation of insulin action. Diabetologia 47:170-184

4. Firth RG, Bell PM, Marsh HM, Hansen I, Rizza RA (1986) Postprandial hyperglycemia in patients with noninsulin-dependent diabetes-mellitus - role of hepatic and extrahepatic tissues. J Clin Investig 77:1525-1532

5. Biddinger SB, Kahn CR (2006) From mice to men: insights into the insulin resistance syndromes. Annu Rev Physiol 68:123-158

6. Abdul-Ghani MA, DeFronzo RA (2010) Pathogenesis of insulin resistance in skeletal muscle. J Biomed Biotechnol 2010: 476279-476295

7. Stefan N, Haring HU (2013) The role of hepatokines in metabolism. Nat Rev Endocrinol 9:144-152

8. Hara H, Yoshimura H, Uchida S et al (2001) Molecular cloning and functional expression analysis of a cDNA for human hepassocin, a liver-specific protein with hepatocyte mitogenic activity. Biochim Biophys Acta Gene Struct Expr 1520:45-53

9. Yu HT, Yu MA, Li CY et al (2009) Specific expression and regulation of hepassocin in the liver and down-regulation of the correlation of HNF1 alpha with decreased levels of hepassocin in human hepatocellular carcinoma. J Biol Chem 284:13335-13347

10. Gao M, Zhan YQ, Yu M et al (2014) Hepassocin activates the EGFR/ERK cascade and induces proliferation of L02 cells through the Src-dependent pathway. Cell Signal 26:2161-2166
11. Wu HT, Lu FH, Ou HY et al (2013) The role of hepassocin in the development of non-alcoholic fatty liver disease. J Hepatol 59: $1065-1072$

12. Liu ZL, Ukomadu C (2008) Fibrinogen-like protein 1, a hepatocyte derived protein is an acute phase reactant. Biochem Biophys Res Commun 365:729-734

13. Lu FH, Ou HY, Wu HT et al (2014) Serum hepassocin concentrations in diabetic patients with or without nonalcoholic fatty liver disease. Diabetes Manag 4:255-261

14. Ohkura T, Shiochi H, Fujioka Y et al (2013) 20/(fasting C-peptide $\times$ fasting plasma glucose) is a simple and effective index of insulin resistance in patients with type 2 diabetes mellitus: a preliminary report. Cardiovasc Diabetol 12:21

15. Ou HY, Wu HT, Hung HC, Yang YC, Wu JS, Chang CJ (2013) Multiple mechanisms of GW-9508, a selective G protein-coupled receptor 40 agonist, in the regulation of glucose homeostasis and insulin sensitivity. Am J Physiol Endocrinol Metab 304: E668-E676

16. Wu HT, Chang CK, Tsao CW et al (2009) Insulin resistance without obesity induced by cotton pellet granuloma in mice. Lab Investig 89:362-369

17. Ayala JE, Bracy DP, Malabanan C et al (2011) Hyperinsulinemiceuglycemic clamps in conscious, unrestrained mice. J Vis Exp 57, e3188

18. Kiechl S, Wittmann J, Giaccari A et al (2013) Blockade of receptor activator of nuclear factor-kappa B (RANKL) signaling improves hepatic insulin resistance and prevents development of diabetes mellitus. Nat Med 19:358-363

19. Williams KH, Shackel NA, Gorrell MD, McLennan SV, Twigg SM (2013) Diabetes and nonalcoholic fatty liver disease: a pathogenic duo. Endocr Rev 34:84-129

20. Hanley AJ, Williams K, Festa A et al (2004) Elevations in markers of liver injury and risk of type 2 diabetes: the insulin resistance atherosclerosis study. Diabetes 53:2623-2632

21. Stefan N, Kantartzis K, Haring HU (2008) Causes and metabolic consequences of fatty liver. Endocr Rev 29:939-960

22. Roskoski R (2012) ERK1/2 MAP kinases: structure, function, and regulation. Pharmacol Res 66:105-143

23. Jiao P, Feng B, Li YJ, He Q, Xu HY (2013) Hepatic ERK activity plays a role in energy metabolism. Mol Cell Endocrinol 375:157-166

24. Kamei N, Tobe K, Suzuki R et al (2006) Overexpression of monocyte chemoattractant protein-1 in adipose tissues causes macrophage recruitment and insulin resistance. J Biol Chem 281: 26602-26614

25. Demchev V, Malana G, Vangala D et al (2013) Targeted deletion of fibrinogen like protein 1 reveals a novel role in energy substrate utilization. Plos One 8, e58084

26. Su YC, Ou HY, Wu HT et al (2015) Prothymosin-alpha overexpression contributes to the development of insulin resistance. J Clin Endocrinol Metab 100:4114-4123 